$5-1-1988$

\title{
Cayley-Tree Ising Model with Antiferromagnetic Nearest-Neighbor and Ferromagnetic Equivalent-Neighbor Interactions
}

\author{
Miron Kaufman \\ Cleveland State University, m.kaufman@csuohio.edu
}

M. Kahana

Follow this and additional works at: https://engagedscholarship.csuohio.edu/sciphysics_facpub

Part of the Physics Commons

How does access to this work benefit you? Let us know!

Publisher's Statement

Copyright 1988 American Physical Society. Available on publisher's site at http://link.aps.org/ doi/10.1103/PhysRevB.37.7638.

\section{Original Citation}

Kaufman, Miron and M. Kahana. "Cayley-Tree Ising Model with Antiferromagnetic Nearest-Neighbor and Ferromagnetic Equivalent-Neighbor Interactions." Physical Review B 37 (1988): 7638-7642.

\section{Repository Citation}

Kaufman, Miron and Kahana, M., "Cayley-Tree Ising Model with Antiferromagnetic Nearest-Neighbor and Ferromagnetic Equivalent-Neighbor Interactions" (1988). Physics Faculty Publications. 81.

https://engagedscholarship.csuohio.edu/sciphysics_facpub/81

This Article is brought to you for free and open access by the Physics Department at EngagedScholarship@CSU. It has been accepted for inclusion in Physics Faculty Publications by an authorized administrator of EngagedScholarship@CSU. For more information, please contact library.es@csuohio.edu. 


\title{
Cayley-tree Ising model with antiferromagnetic nearest-neighbor and ferromagnetic equivalent-neighbor interactions
}

\author{
Miron Kaufman and Michael Kahana \\ Department of Physics, Cleveland State University, Cleveland, Ohio 44115
}

(Received 19 November 1987)

\begin{abstract}
The phase diagram of the Ising model with antiferromagnetic nearest-neighbor interactions and ferromagnetic equivalent-neighbor interactions on the Cayley tree is determined exactly. A nonuniversal critical line separates the disordered and the ordered phases. A line of first-order transitions separating ferromagnetic order from antiferromagnetic order ends in the midst of the ordered phase at a classical ordered critical point. For a small range of values of the ratio of the two couplings, two transitions occur as the temperature is varied. In this case the uniform magnetization is not a monotonic function of the temperature.
\end{abstract}

\section{INTRODUCTION}

Dynamical and equilibrium properties of disordered systems have been the object of studies using hierarchical models. Hierarchical structures used to model the dynamics of glassy materials ${ }^{1}$ displayed the experimentally observed Kohlrausch relaxation law and the VogelFulcher law. Mode condensation was also analyzed ${ }^{2}$ on a hierarchical tree structure. Berker and collaborators ${ }^{3}$ used frustrated hierarchical lattices to study spin glasses in equilibrium. Kardar and Kaufman showed for Ising, ${ }^{4}$ percolation, and Potts ${ }^{5}$ systems that turning on ferromagnetic interactions of Cayley-tree connectivity in the equivalent-neighbor model has an effect similar to lowering the spatial dimension. This is reminiscent of the dimensional reduction effect ${ }^{6}$ in another disordered system: the random-field Ising model.

Cayley trees are hierarchical lattices ${ }^{7}$ which generally lack translational invariance. Since statistical models are amenable to exact solutions on hierarchical lattices, it has been suggested ${ }^{8}$ that such studies could contribute to our understanding of disordered and other low-symmetry systems. In this paper we solve the Cayley-tree Ising model with antiferromagnetic nearest-neighbor and ferromagnetic equivalent-neighbor interactions. It is important to point from the outset that, in contrast to the BethePeierls approximation, the boundary of the tree is included in the calculation. The large boundary (finite fraction of the total number of degrees of freedom) influences the thermodynamics of the system. Boundary inclusion generates a system that lacks translational invariance. Thus, while the equivalent-neighbor couplings preserve the equivalence of all degrees of freedom, the turning on of Cayley-tree couplings breaks this symmetry.

The phase diagram and the critical behavior of this system is determined by three competitions: (i) infinite range versus short range; (ii) preservation of translational invariance versus hierarchical breaking of it; (iii) ferromagnetic versus antiferromagnetic. As a consequence of competitions (i) and (ii) a nonuniversal critical line separates the disordered and the ordered phases. This feature was also observed ${ }^{4,5}$ when the Cayley-tree cou- pling was ferromagnetic. For small values of the nearest-neighbor coupling the critical exponents are classical, while for large values of this coupling the exponents are nonclassical and change continuously with the coupling. The two regimes are separated at a special value of the nearest-neighbor coupling by classical criticality modified by logarithms. Thus increasing the strength of the Cayley-tree coupling has an effect similar to lowering the spatial dimension.

A novel feature presented in this paper is the occurrence of a line of first-order transitions separating ferromagnetic and antiferromagnetic phases. This line ends at a critical point in the midst of the ordered phase. This critical entity, which we call ordered critical point, also occurs in other spin models such as the spin- $\frac{3}{2}$ model and the trimodal random-field Ising model. ${ }^{9}$ There are thermodynamic paths connecting the two phases without crossing any phase boundary. This unusual feature is rooted in the inhomogeneous character of the tree which makes the order parameter to be spatially varying.

A special case of the model studied here, the antiferromagnetic one-dimensional (Cayley tree of coordination number $z=2$ ) Ising model with ferromagnetic equivalent-neighbor interactions, was studied before by Nagle $^{10}$ and Kardar. ${ }^{11}$ Nagle's motivation for studying the model was the hypothesis that two transitions can occur as the temperature is varied. Though this expectation is not realized for $z=2$, it is interesting to point out that for trees of coordination number $z \geq 3$, two transitions can occur as the temperature is lowered: first a continuous transition from the disordered to the ordered phase, and then a discontinuous transition between antiferromagnetic and ferromagnetic phases. In this case the uniform magnetization is not a monotonic function of temperature.

The remainder of this paper is organized as follows. In Sec. II the analytical formulae necessary to compute the free energy are derived. In Sec. III the critical behavior along the critical line separating the ordered and disordered phases is determined. Section IV contains our numerical analysis of the ordered phase. A summary of our results can be found in Sec. V. 


\section{MODEL AND SOLUTION}

Ising spins $s= \pm 1$ are located at the vertices of the Cayley tree of coordination number $z$. All pairs of neighboring spins are connected by antiferromagnetic bonds $K<0$, and all pairs of spins irrespective of their location are connected by ferromagnetic bonds $J>0$. The Hamiltonian is

$$
-\frac{\mathscr{H}}{k T}=\frac{J}{2 N} \sum_{i, j} s_{i} s_{j}+K \sum_{\langle i, j\rangle} s_{i} s_{j},
$$

where $N$ is the total number of spins. The partition function is

$$
Z=\sum_{\left\{s_{i}\right\}} \exp \left(-\frac{\mathscr{H}}{k T}\right) .
$$

To compute the partition function we follow Kardar's Hamiltonian minimization method. ${ }^{11}$ By using a Gaussian integral, $Z$ can be written as

$$
\begin{aligned}
Z=(N J / 2 \pi)^{1 / 2} \int_{-\infty}^{\infty} d m \exp [ & -\frac{N}{2} J m^{2}+J m \sum_{i} s_{i} \\
& \left.+K \sum_{\langle i, j\rangle} s_{i} s_{j}\right) .
\end{aligned}
$$

After summing over the spin configurations in the righthand side of Eq. (3) we find

$$
Z=\int_{-\infty}^{\infty} d m \exp \left\{-N\left[\frac{1}{2} J m^{2}+f_{0}(K, J m)\right]\right\},
$$

where terms of order $\ln N / N$ in the exponent were neglected, and $f_{0}(K, J m)$ is the free energy of the Cayley-tree model with a coupling $K$ and a uniform field equal to $\mathrm{Jm}$. In the thermodynamic limit $N \rightarrow \infty$ the free energy per spin $f=-\ln Z / N$ is determined by the largest integrand on the right-hand side of Eq. (4):

$$
f=\min _{m} \psi(m) \text { and } \psi(m)=\frac{1}{2} J m^{2}+f_{0}(K, J m) .
$$

The value of $m$ which minimizes $\psi(m)$ is the uniform magnetization: ${ }^{11} m=\Sigma_{i} s_{i} / N$.

The free energy of the Cayley-tree Ising model in a field $h$ is obtained by successively decimating the Ising spins starting from the boundary of the tree

$$
\begin{aligned}
-f_{0}=\frac{1}{2} \frac{z-2}{z-1} \sum_{n=0}^{\infty}(z-1)^{-n} \ln [ & 2 \cosh (2 K) \\
& \left.+2 \cosh \left(2 h_{n}\right)\right],
\end{aligned}
$$

where $h_{n}$ is the effective field acting on spins on the $n$th tree generation and which is given by the following recursion equation:

$$
h_{n}=h+\frac{z-1}{2} \ln \frac{\cosh \left(K+h_{n-1}\right)}{\cosh \left(K-h_{n-1}\right)} \text {, }
$$

and

$$
h_{0}=h \text {. }
$$

The free energy $f_{0}$ is a nonanalytical function of $h$ for $h \rightarrow 0$ provided $|K|>K_{\mathrm{BP}}$ where $K_{\mathrm{BP}}$ is the BethePeierls value of the coupling: $\tanh K_{\mathrm{BP}}=1 /(z-1)$. The free-energy expansion in powers of $h$ is ${ }^{12}$

$$
-f_{0}=\sum_{n=0}^{\infty} \frac{\chi_{2 n}}{(2 n) !} h^{2 n}+A|h|^{\Delta} .
$$

For $|K|<K_{\mathrm{BP}} f_{0}$ is an analytical function of $h$, and $A=0$ in Eq. (8). The exponent $\Delta$ is determined by standard renormalization-group arguments from the recursion Eq. (7):

$$
\Delta=\frac{\ln (z-1)}{\ln [(z-1) \tanh |K|]} .
$$

When $\Delta=2 m$ (even positive integer) the power singularity in Eq. (8) is replaced by a logarithmic singularity $f_{0} \sim h^{2 m} \ln |h|$. The source ${ }^{13}$ of the logarithmic singularity is the breaking down of the Taylor expansion of the regular part of $f_{0}$.

\section{CRITICAL BEHAVIOR}

At sufficiently high temperatures, i.e., small $J$ and $|K|$, the system is disordered and the uniform magnetization is $m=0$. At sufficiently low temperatures, i.e., large $J$ and $|K|, \psi(m)$ as defined in Eq. (5) is minimized at $m \neq 0$ and this is the ordered phase. To determine the line of critical points separating the ordered and disordered phases and the critical exponents, we expand $\psi$, Eq. (5), in powers of $m$ by using Eq. (8):

$$
\begin{aligned}
\psi(m)= & -\chi_{0}+\frac{J}{2 !}\left(1-J \chi_{2}\right) m^{2} \\
& -\frac{\chi_{4}}{4 !} J^{4} m^{4} \ldots-A J^{\Delta}|m|^{\Delta} .
\end{aligned}
$$

Criticality is achieved when the quadratic coefficient vanishes:

$$
\begin{aligned}
J & =\frac{1}{\chi_{2}} \\
& =\left[1-(z-1) \tanh ^{2} K\right] /[1+\tanh K]^{2} \text { for }|K|<K_{2},
\end{aligned}
$$

and

$$
J=0 \text { for }|K|>K_{2} \text {, }
$$

where $\tanh K_{2}=(z-1)^{-1 / 2}$ and $\chi_{2}$ was first derived by Heimburg and Thomas. ${ }^{14}$

The critical exponents are determined by the same analysis as that performed ${ }^{4}$ for positive $K$. For $|K|<K_{4}$, where $\tanh K_{4}=(z-1)^{-3 / 4}$, the exponent $\Delta>4$, see Eq. (9), and the asymptotic behavior is determined by the $m^{2}$ and $m^{4}$ contributions to $\psi(m)$. The quartic coefficient is positive because $\chi_{4}<0 .{ }^{15}$ The critical exponents are classical: $\alpha=0$ and $\beta=\frac{1}{2}$. For $K_{4}<|K|<K_{2}$ the asymptotic behavior is determined by the $m^{2}$ and $|m|^{\Delta}$ terms because $2<\Delta<4$ and $A<0$. The critical exponents are now nonclassical and 
nonuniversal: $\alpha=-(4-\Delta) /(\Delta-2)$ and $\beta=1 /(\Delta-2)$. The two regimes of classical and nonclassical criticality are separated at $|K|=K_{4}$ or $\Delta=4$ by classical behavior modified by logarithms. ${ }^{4}$ At $|K|=K_{2}$ or $\Delta=2$ the thermodynamic functions exhibit exponential singularities. ${ }^{4}$ Thus $\Delta$ plays a role similar to the spatial dimension and the special values 2 and 4 correspond to the lower- and upper-critical dimensions, respectively. For $|K|>K_{2}$ the exponent $\Delta<2$ and $\psi(m)$ given in Eq. (10) can be approximated by

$$
\psi(m)=-\chi_{0}-A J^{\Delta}|m|^{\Delta}+J m^{2} / 2,
$$

where $A<0$. As $J \rightarrow 0$ the magnetization vanishes as $m \sim J^{\beta}$, where $\beta=(\Delta-1) /(2-\Delta)$, and $\partial^{2} f / \partial J^{2} \sim J^{-\alpha}$, where $\alpha=(4-3 \Delta) /(2-\Delta)$.

\section{ORDERED PHASE}

At large values of the couplings $J$ and $|K|$ long-range order is established. At zero temperature, $J \rightarrow \infty$ and $-K \rightarrow \infty$, two types of order occur. The ferromagnetic order, with all spins equal to each other $(m= \pm 1)$, occurs when the ferromagnetic coupling $J$ is large compared to the antiferromagnetic interaction. The antiferromagnetic order is characterized by spins on adjacent tree generations taking opposite values, but with all spins of a given generation having the same value. The uniform magnetization is $m= \pm(z-2) / z$ and this phase occurs when the antiferromagnetic strength $|K|$ is large compared to the ferromagnetic coupling. The two phases are separated by a first-order transition which is determined by balancing the ferromagnetic and antiferromagnetic energies. The ground-state energies for the Cayley tree in a field $h$ are $-f_{0}=K \pm h$ for ferromagnetic order and $-f_{0}=-K$ $\pm h(z-2) / z$ for antiferromagnetic order. The minimization of $\psi(m)$, after substituting the $f_{0}$ formula into Eq. (5), yields $m= \pm 1$, and $m= \pm(z-2) / z$. The corresponding energies are equal if

$$
J=-K z^{2} /(z-1) \text {. }
$$

The phase diagram in the plane $(K, J)$ was determined numerically by searching for the minimum of $\psi(m)$ given in Eq. (5), after computing the free energy $f_{0}$ of the nearest-neighbor model in a uniform field $h=J m$ from Eqs. (6) and (7). Figure 1 contains the phase diagram for a tree of coordination number $z=3$. The positive $K$ region ${ }^{4}$ is also shown. The line of first-order transitions which in the zero-temperature limit is given by Eq. (12) $J=-4.50 \mathrm{~K}$, ends at an ordered critical point: $-K=0.7514, J=3.417$ in the midst of the ordered phase. This is a classical (mean-field) multicritical point. Figure 2 shows the temperature dependence along the first-order transitions line of the difference of the uniform magnetization values from each side of the line, and the temperature is defined as $T=1 /(k J)$. At $T=0$, this difference equals $1-(z-2) / z=2 / z$ and it vanishes at the ordered critical point with an exponent $\beta$ equal to the mean-field value of $\frac{1}{2}$. The magnetization value at the ordered critical point is $m=0.61$.

Note that the ferromagnetic and antiferromagnetic re- gions of the phase diagram can be joined by certain paths which do not cross the first-order transitions line, in contradiction with the expectations one has for a Bravais lattice system. The solution of this apparent paradox lies in the nonuniform character of the Cayley tree. In the ferromagnetic region the average magnetization changes from tree generation to tree generation. Also, unlike for a square or cubic lattice, there is a nonvanishing uniform magnetization in the antiferromagnetic region. Thus, each of these phases exhibits nonvanishing values of the

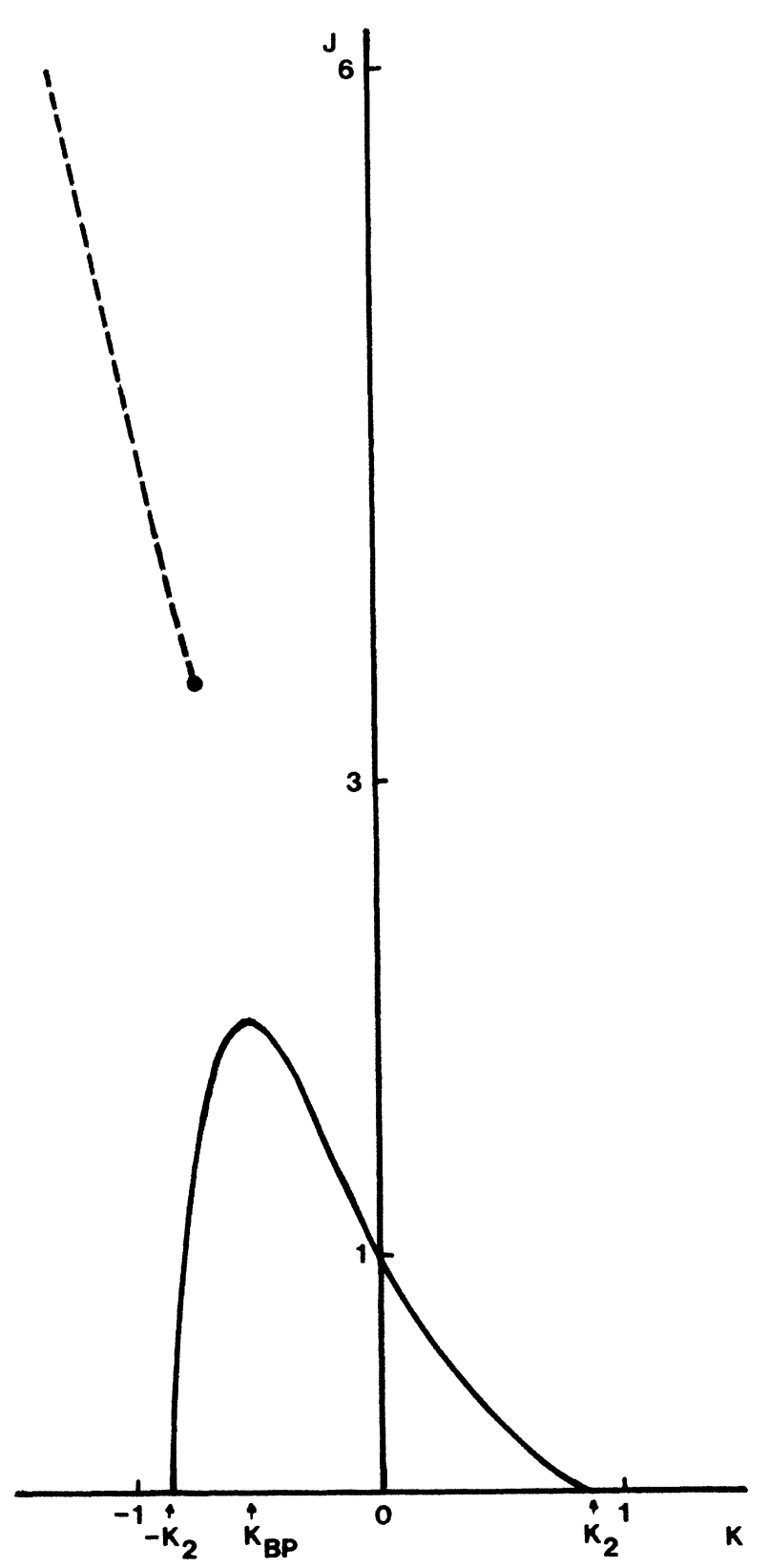

FIG. 1. Phase diagram in the plane $(K, J)$ for the tree of coordination number $z=3$. The dashed line is a line of firstorder transitions which ends at an ordered critical point. The solid line is a line of nonuniversal second-order transitions. It has a maximum at $K=-K_{\mathrm{BP}}, J=(z-1) /(z-2)=2$, and it intersects the $K$ axis at $K= \pm K_{2}$. 


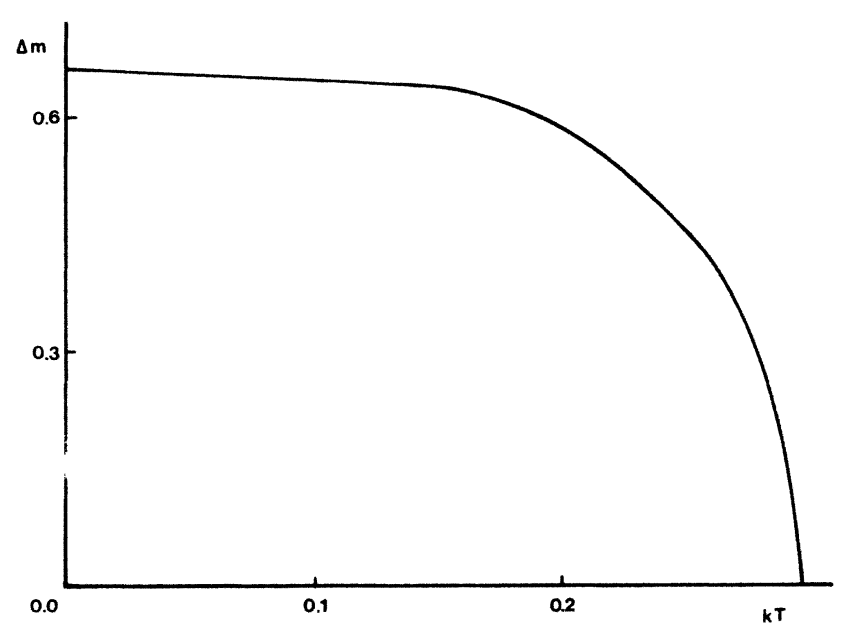

FIG. 2. The temperature $(k T=1 / J)$ dependence along the first-order transitions line of the difference $\Delta m$ of magnetizations values from each side of the line, for the tree of coordination number $z=3$. At $T=0, \Delta m=2 / z=\frac{2}{3}$, and close to the ordered critical point $\Delta m \sim|t|^{1 / 2}$, where $t$ is the reduced temperature.

order parameter of the other phase. A better terminology could be "predominantly ferromagnetic" and "predominantly antiferromagnetic" phases, rather than ferromagnetic and antiferromagnetic phases. One can imagine changing the properties of the system smoothly and moving from one regime to the other without crossing a phase boundary.

The phase diagram remains topologically unchanged for any coordination number $3 \leq z<\infty$. In the limit $z \rightarrow \infty$ the disordered region of the phase diagram shrinks to the segment $K=0, \quad J<1$, because $\tanh K_{2}$

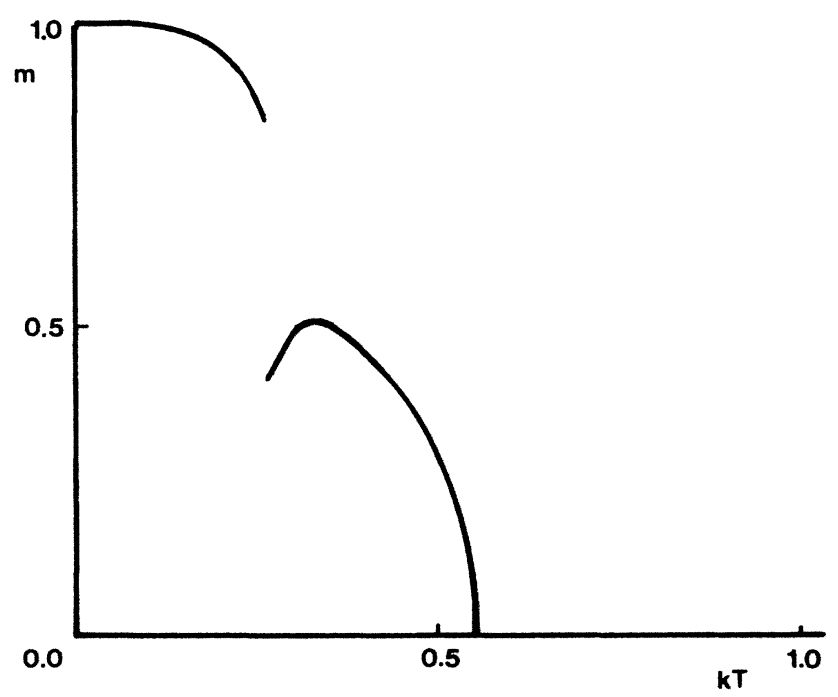

FIG. 3. The temperature $(k T=1 / J)$ dependence of the magnetization $m$ for $J / K=-4.52$, for the tree of coordination number $z=3$. As the temperature is increased two phase boundaries are crossed: first a discontinuous transition and then a classical continuous transition. The magnetization is not monotonically decreasing as the temperature is increased. $=(z-1)^{-1 / 2} \rightarrow 0$. The first-order transitions line disappears as the distinction between antiferromagnetic and ferromagnetic order vanishes: $m=(z-2) / z \rightarrow 1$. For $z=2$ the Cayley tree reduces to the one-dimensional lattice, and the phase diagram ${ }^{10,11}$ differs topologically from Fig. 1. The critical line of Eq. (11) is now $J=(1-\tanh K) /(1+\tanh K)=\exp (-2 \mathrm{~K})$. It intersects the first-order transitions line, which in the zerotemperature limit is $J=-4 \mathrm{~K}$ at a tricritical point ${ }^{10,11}$ located at $-K=(\ln 3) / 4, J=3^{1 / 2}$.

The first-order transitions segment, though very close to a straight line in the plane $(K, J)$, is actually curved. Consequently, there exists a small interval $4.50<J /|K|<4.55$ for which two transitions occur as the temperature $T=1 /(k J)$, is lowered: first a classical continuous transition from the disordered to the ordered phase; then a discontinuous transition between antiferromagnetic and ferromagnetic phases. Figure 3 which con-
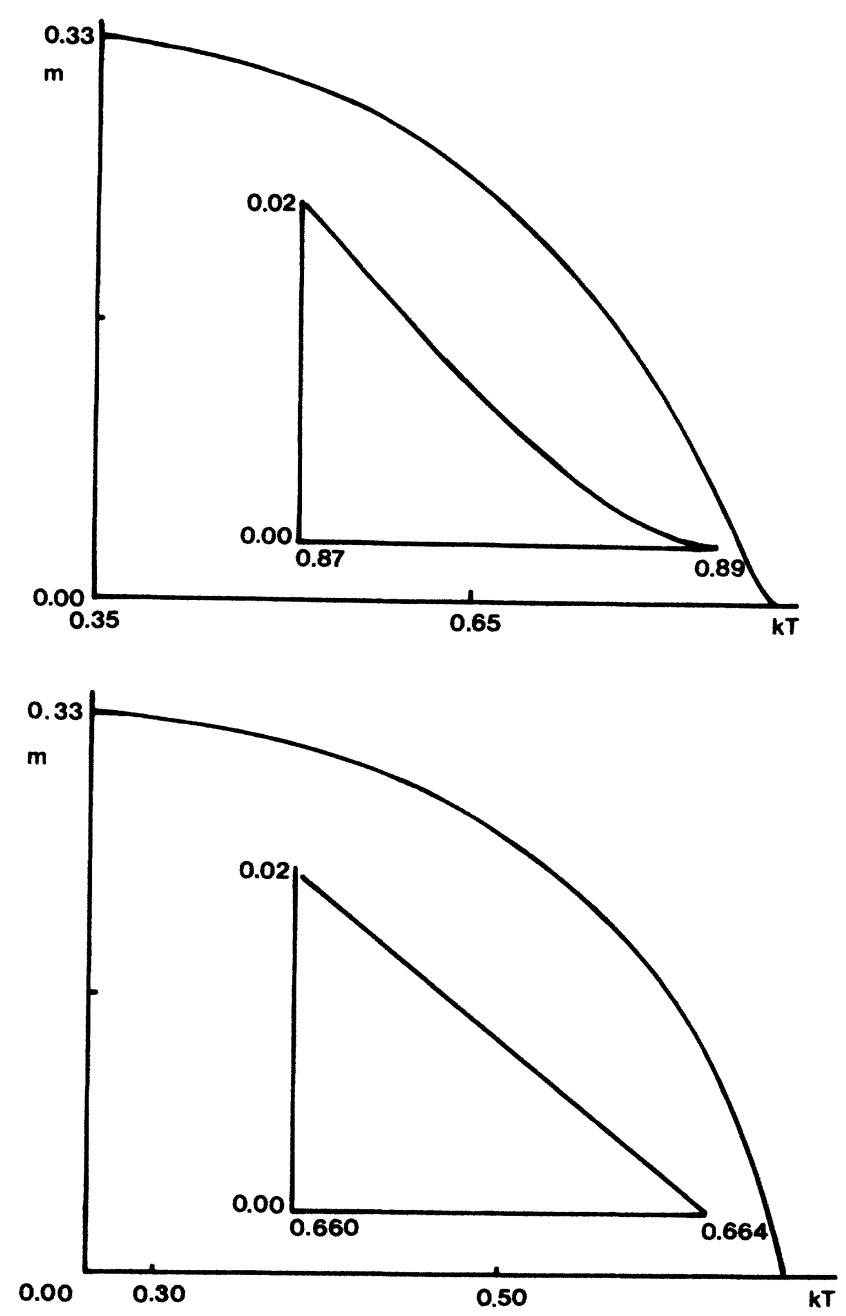

FIG. 4. The temperature $(k T=1 / J)$ dependence of the magnetization $m$ for $J / K=-1.4112$ and -2.0322 , respectively, for the tree of coordination number $z=3$. Nonclassical critical behavior is observed close to the critical points, reduced temperature $|t|<10^{-3}$, as shown in the insets. The corresponding values of the exponent $\beta$ are 2 and 1, respectively. 
tains the temperature dependence of the uniform magnetization for $J /|K|=4.52$, shows the two transitions. We note that the search for two transitions was a motivation for Nagle's study ${ }^{10}$ of the one-dimensional problem, i.e., $z=2$. Two transitions occur for trees of coordination number $z \geq 3$. As it is apparent from Fig. 3 the uniform magnetization $m$ is not a monotonic function of the temperature. This is not a violation of any Griffiths, Kelley, and Sherman (GKS) correlation inequality ${ }^{16}$ as these inequalities hold for ferromagnetic models while here antiferromagnetic couplings are also present. Nonmonotonic temperature dependence of the magnetization was observed earlier in ferromagnetic superconductors. ${ }^{17}$

We also verified numerically the exponents derived theoretically in Sec. III. In Fig. 4 the temperature dependence of the uniform magnetization is shown for $J /|K|=1.4112$ and 2.0322 , respectively. The corresponding values of the critical $K$ substituted in Eq. (9) yield $\Delta=2.5$ and 3 , respectively. The respective critical exponent $\beta$ values are 2 and 1 (see Sec. III). The theoretical critical exponent values and the theoretical location of the critical points are corroborated by the numerical results.

\section{SUMMARY}

The Ising model with antiferromagnetic nearestneighbor interactions and ferromagnetic equivalentneighbor interactions on the Cayley tree was solved exactly. This model exhibits three types of competitions: short-range versus infinite-range interactions, hierarchical breaking of versus preservation of translational symmetry, and antiferromagnetic versus ferromagnetic in- teractions. The competition of ferromagnetic and antiferromagnetic couplings in the interior of Cayley trees was studied by Thouless and collaborators ${ }^{18}$ within the Bethe lattice spin-glass model. The latter is an inhomogeneous system due to quenched randomness. In our model, on the other hand, the system is inhomogeneous due to the inclusion of the thermodynamically sized boundary of the tree.

The phase diagram includes a nonuniversal critical line separating the disordered and ordered phases. Inside the ordered phase a line of first-order transitions separates ferromagnetic and antiferromagnetic types of order. This line ends in the midst of the ordered phase at an ordered critical point. Thus the two types of order can be joined by thermodynamic paths which do not cross any phase boundary. Such a situation cannot arise on a square or cubic lattice, and it is due to the absence of translational invariance (spatially varying order parameter) on Cayley trees with boundaries. For a certain range of values of the ratio of the two couplings, two transitions occur as the temperature is changed, and the uniform magnetization is not a monotonic function of the temperature. Successive transitions as the temperature is varied were recently observed $^{19}$ in the kagomé lattice Ising model with two competing couplings.

\section{ACKNOWLEDGMENTS}

We have benefited from discussions with Mehran Kardar and correspondence with John Nagle. This work was supported by the Ohio Board of Regents through a Research Challenge Grant.
${ }^{1}$ R. G. Palmer, D. L. Stein, E. Abrahams, and P. W. Anderson, Phys. Rev. Lett. 53, 958 (1984); A. T. Ogielsky and D. L. Stein, ibid. 55, 1634 (1985); S. Teitel, D. Kutasov, and E. Domany, Phys. Rev. B 36, 684 (1987); T. A. Vilgis, J. Stat. Phys. 47, 133 (1987).

${ }^{2}$ D. Kumar and Kamlesh, Phys. Rev. Lett. 57, 3101 (1986).

${ }^{3}$ S. R. McKay, A. N. Berker, and S. Kirkpatrick, Phys. Rev. Lett. 48, 767 (1982); J. Appl. Phys. 53, 7974 (1982); S. R. McKay and A. N. Berker, Phys. Rev. B 29, 1315 (1984); J. Appl. Phys. 55, 1646 (1984).

${ }^{4}$ M. Kardar and M. Kaufman, Phys. Rev. Lett. 51, 1210 (1983).

${ }^{5}$ M. Kaufman and M. Kardar, Phys. Rev. B 29, 5053 (1984); 30, 1609 (1984).

${ }^{6}$ A. Aharony, Y. Imry, and S.-K. Ma, Phys. Rev. Lett. 37, 1364 (1976).

${ }^{7}$ A. N. Berker and S. Ostlund, J. Phys. C 12, 4961 (1979); R. B. Griffiths and M. Kaufman, Phys. Rev. B 26, 5022 (1982); M. Kaufman and R. B. Griffiths, ibid. 30, 244 (1984); A. N. Berker and S. R. McKay, J. Stat. Phys. 36, 787 (1984); H. Moraal, Vol. 214 of Lecture Notes in Physics, (Springer-Verlag, New York, 1984); B. Hu, Phys. Rev. Lett. 55, 2316 (1985).

${ }^{8}$ M. Kaufman and R. B. Griffiths, Phys. Rev. B 24, 496 (1981).

${ }^{9}$ S. Krinsky and D. Mukamel, Phys. Rev. B 11, 399 (1975); M.
Kaufman, P. E. Klunzinger, and A. Khurana, ibid. 34, 4766 (1986).

10J. F. Nagle, Phys. Rev. A 2, 2124 (1970); J. F. Nagle and J. C. Bonner, J. Chem. Phys. 54, 729 (1971).

${ }^{11}$ M. Kardar, Phys. Rev. Lett. 51, 523 (1983); Phys. Rev. B 28, 244 (1983).

${ }^{12}$ E. Müller-Hartmann and J. Zittartz, Phys. Rev. Lett. 33, 893 (1974); E. Müller-Hartmann, Z. Phys. B 27, 161 (1977).

${ }^{13}$ M. Kaufman and D. Andelman, Phys. Rev. B 29, 4010 (1984); S. I. Chase and M. Kaufman, ibid. 33, 239 (1986); M. Kaufman, ibid. 36, 3697 (1987).

${ }^{14}$ J. von Heimburg and H. Thomas, J. Phys. C 7, 3433 (1974).

${ }^{15}$ H. Falk, Phys. Rev. B 12, 5184 (1975); T. Morita and T. Horiguchi, J. Stat. Phys. 26, 665 (1981).

${ }^{16}$ R. B. Griffiths, J. Math. Phys. 7, 478 (1967); 8, 484 (1967); D. G. Kelly and S. Sherman, ibid. 9, 466 (1968).

${ }^{17}$ M. Kaufman and O. Entin-Wohlman, Physica B 84, 90 (1976).

${ }^{18}$ D. J. Thouless, Phys. Rev. Lett. 56, 1082 (1986); J. M. Carlsson, J. T. Chayes, L. Chayes, J. P. Sethna, and D. J. Thouless (unpublished).

${ }^{19}$ P. Azaria, H. T. Diep, and H. Giacomini, Phys. Rev. Lett. 59, 1629 (1987). 